\title{
An Essential Quality Control Mechanism at the Eukaryotic Basal Body Prior to Intraflagellar Transport
}

\author{
Angela Stephan ${ }^{1, \dagger}$, Sue Vaughan ${ }^{2, *, \dagger}$, \\ Michael K. Shaw ${ }^{2}$, Keith Gull ${ }^{2}$ and \\ Paul G. McKean ${ }^{1, *}$ \\ ${ }^{1}$ Biomedical Sciences Unit, Department of Biological \\ Sciences, Lancaster University, Lancaster, \\ LA1 4YQ, UK \\ ${ }^{2}$ Sir William Dunn School of Pathology, University of \\ Oxford, South Parks Road, Oxford, OX1 3RE, UK \\ ${ }^{*}$ Corresponding author: Paul G. McKean, \\ p.mckean@lancaster.ac.uk or Sue Vaughan, \\ susan.vaughan@path.ox.ac.uk \\ tThese authors contributed equally to this work.
}

Constructing a eukaryotic cilium/flagellum is a demanding task requiring the transport of proteins from their cytoplasmic synthesis site into a spatially and environmentally distinct cellular compartment. The clear potential hazard is that import of aberrant proteins could seriously disable cilia/flagella assembly or turnover processes. Here, we reveal that tubulin protein destined for incorporation into axonemal microtubules interacts with a tubulin cofactor $\mathrm{C}$ (TBCC) domain-containing protein that is specifically located at the mature basal body transitional fibres. RNA interference-mediated ablation of this protein results in axonemal microtubule defects but no effect on other microtubule populations within the cell. Bioinformatics analysis indicates that this protein belongs to a clade of flagellum-specific TBCC-like proteins that includes the human protein, XRP2, mutations which lead to certain forms of the hereditary eye disease retinitis pigmentosa. Taken with other observations regarding the role of transitional fibres in cilium/flagellum assembly, we suggest that a localized protein processing capacity embedded at transitional fibres ensures the 'quality' of tubulin imported into the cilium/flagellum, and further, that loss of a ciliary/flagellar quality control capability may underpin a number of human genetic disorders.

Key words: basal body, chaperone, flagellum, intraflagellar transport, retinitis pigmentosa, tubulin, tubulin cofactor C, trypanosome

Received 7 March 2007, revised and accepted for publication 8 June 2007, uncorrected manuscript published online 11 June 2007, published online 20 July 2007

Eukaryotic cilia and flagella are evolutionarily conserved organelles that perform a diversity of biological functions, ranging from motility to sensory perception and are vital to human health. Defects in cilium/flagellum function underpin a wide range of inherited human disorders including respiratory disease, retinal degeneration as well as more complex developmental disorders such as Bardet-Biedl syndrome (BBS) and hydrocephalus (1). The construction of a flagellum/cilium, involving assembly of several hundred distinct proteins (2-5), is made more difficult by the fact that in most eukaryotes, assembly occurs in a ribosome-free cellular compartment distinct, and ultimately distant, from the normal cellular cytoplasm (6). This assembly mechanism provides additional challenges and necessitates accurate intracellular targeting, processing and transport of proteins into the cilium/flagellum.

With a few exceptions $(7,8)$, cilium/flagellum assembly relies upon an evolutionarily conserved bi-directional transport mechanism known as intraflagellar transport (IFT) (reviewed in 9). Transitional fibres radiating from the mature basal body demarcate the boundary of the cilium/ flagellum compartment and are pivotal to this process acting as both docking sites for IFT motor proteins and regulating entry of IFT particles into the cilium/flagellum $(10,11)$. Molecular understanding of IFT has advanced rapidly in recent years, yet our knowledge of events relating to recruitment and processing of proteins prior to IFT-mediated translocation remains poor.

The major structural component of all eukaryotic cilia/ flagella (whether motile or sensory) is a microtubule-based axoneme constructed from the heterodimeric protein, $\alpha / \beta$-tubulin. The generation of a functional $\alpha / \beta$-tubulin heterodimer depends upon a well-defined tubulin-folding pathway, in which quasi-native intermediates sequentially interact with specific tubulin cofactors (TBCC) (12-15). Functional $\alpha / \beta$-tubulin heterodimers are ultimately generated from an interaction between TBCC and a super-complex containing $\alpha / \beta$-tubulin monomers and tubulin-specific cofactors TBCD and TBCE. This interaction with TBCC stimulates hydrolysis of GTP by $\beta$-tubulin and releases GDP-bound $\alpha / \beta$-tubulin heterodimer; subsequent free exchange results in the production of a GTP-bound tubulin heterodimer competent for microtubule incorporation. The necessity to hydrolyse GTP to enact heterodimer release may function as a quality control step ensuring that only GTPase competent heterodimer is released. This is an important consideration as polymerization of $\alpha / \beta$-tubulin heterodimers into microtubules is intimately coupled to hydrolysis of the GTP nucleotide bound to $\beta$-tubulin. The question therefore arises, as to how such protein quality control processes are handled when tubulin is exported from the cytoplasm into the flagellum compartment.

We have exploited the experimentally tractable flagellated protozoan parasite Trypanosoma brucei (a pathogen of 
significant medical and veterinary importance in Sub-Saharan Africa) to investigate tubulin processing requirements during cilium/flagellum formation. T. brucei possesses a single flagellum and forms a new flagellum during each cell division cycle. Although some ciliated/flagellated cells (e.g. the green algae Chlamydomonas reinhardtii) resorb cilia/flagella during the cell cycle, T. brucei (and many other ciliated/flagellated cells) divide with flagella present. Trypanosomes thus provide a model to compare processes operating to construct and maintain a new and old flagellum, respectively, within the same cell.

This study reveals that a flagellum-specific isoform of TBCC is embedded at transitional fibres radiating from the mature basal body, where it provides an essential cilium/flagellum-specific function. Detailed bioinformatics analyses show that this trypanosome protein belongs to a family of related TBCC domain-containing proteins, whose phylogenetic occurrence is restricted to eukaryotic organisms capable of forming a flagellum/cilium. This clade also contains the human protein XRP2, mutations in which lead to certain forms of the hereditary eye disease $X$-linked retinitis pigmentosa (RP, OMIM 312600), characterized by progressive degeneration of photoreceptor cells in the retina. Within retinal cells, a modified cilium provides an intracellular link between the functionally and morphologically distinct inner and outer photoreceptor compartments (16) and we suggest that our results may provide insight into the molecular basis for both this disease and also a number of other inherited human diseases characterized by dysfunctional cilia.

\section{Results}

\section{Bioinformatics analysis identifies a flagellum/cilium TBCC}

We recently completed a comprehensive proteomic analysis of the T. brucei flagellum resulting in the publication of the T. brucei flagellar proteome (TbFP) (2). Of the 331 proteins present in the TbFP, 86 are present in the genomes of at least one other flagellated/ciliated eukaryote but absent in the genomes of non-flagellates. Given the evolutionary distance between trypanosomes and other flagellated/ciliated eukaryotes, these proteins represent a highly conserved flagellum/cilium-specific cohort. Among this conserved cohort, we identified a T. brucei protein (GeneDB accession number Tb10.61.2870), which encodes a domain with homology to TBCC, one of the key molecular chaperones required to assemble functional $\alpha / \beta$-tubulin heterodimer. As TBCC is likely to have an essential role in tubulin formation in all eukaryotes, identification of this protein as flagellum/cilium specific appeared anomalous.

Using an iterative profile-based searching technique (based on a Pfam TBCC Hidden Markov model seed alignment), we interrogated a wide range of phylogenet- ically diverse eukaryotic organisms to identify all proteins possessing homology with the Pfam-defined TBCC domain (Figure 1). This analysis revealed that many (but intriguingly not all) eukaryotes encode several TBCC proteins that resolve into three distinct clades. Clade 1 contains TBCC proteins from a diverse range of eukaryotes and includes the canonical human and yeast cytoplasmic TBCC protein essential for de novo $\alpha / \beta$-tubulin heterodimer formation. However, our analysis reveals that TBCC domain-containing proteins in clade 2 are restricted to eukaryotes capable of forming a cilium/flagellum and includes human RP2 and the Tb10.61.2870 protein detected in our flagellar proteome (hereafter designated TbRP2). Clade 3 contains proteins from a range of flagellated and non-flagellated eukaryotes. However, the TBCC domain identified in this clade does not easily align with proteins from clades 1 and 2, particularly with regard to a key arginine residue previously identified as critical to TBCC protein function (17), raising questions regarding the functionality of TBCC proteins within this clade. Our analysis also reveals another intriguing anomaly. Despite constructing cilia and/or flagella, the fruit fly Drosophila melanogaster and the apicomplexan parasite Plasmodium falciparum do not encode a flagellate-specific isoform of TBCC. This may simply reflect the different way these organisms build cilia/flagella $(8,18)$.

\section{RNA interference-mediated ablation of TbRP2 results in structurally aberrant flagella}

Biochemical studies have shown that human RP2 protein has partial functional overlap with canonical TBCC, in that RP2 can stimulate the GTPase activity of native tubulin heterodimers but cannot generate $\alpha / \beta$-tubulin heterodimer from $\alpha / \beta$-tubulin monomers (17). Despite this biochemical evidence, there is no direct experimental evidence demonstrating that RP2 mutations lead to microtubule abnormalities and so the in vivo function of RP2 remains a matter of debate (19). To address this question, we subjected the trypanosome protein TbRP2 to functional analysis using RNA interference (RNAi) and demonstrated that induction of RNAi in procyclic (insect stage) trypanosomes resulted in increasingly severe growth defects from $48 \mathrm{~h}$ post-induction (Figure 2A). Real-time reverse transcriptase-polymerase chain reaction experiments show reduction of TbRP2messenger RNA levels to $<10 \%$ of non-induced controls within $48 \mathrm{~h}$ of induction (data not shown).

Trypanosomes possess a single flagellum and construct a new flagellum during each cell cycle. As the new flagellum is invariantly positioned posterior to the old, it is possible to unambiguously distinguish new and old flagella in dividing trypanosomes. Observation of TbRP2RNAi induced trypanosomes reveals that the new flagellum is abnormally short (compare the non-induced cell in Figure 2B with the TbRP2-ablated cell in Figure 2C). Conformation that the length of this new flagellum is aberrant comes from comparison with cell-cycle event markers 


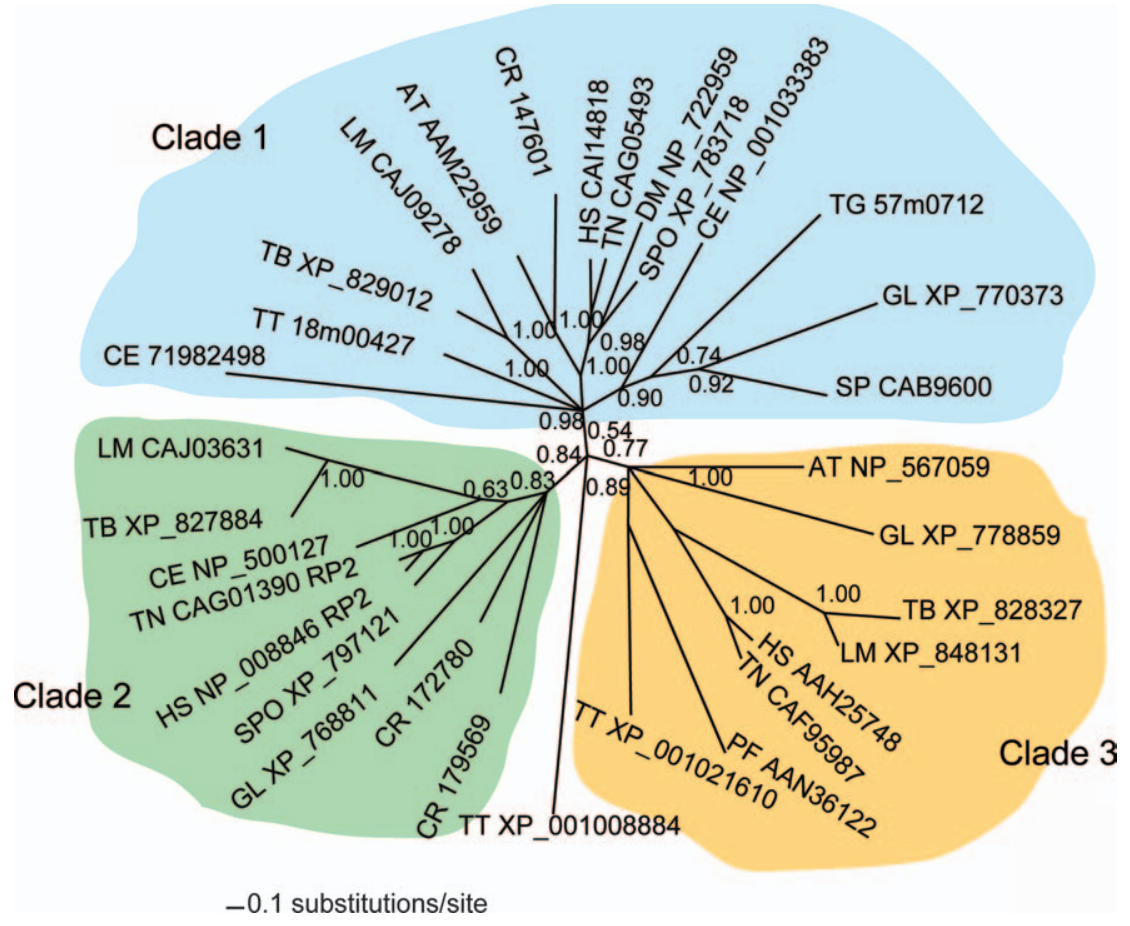

Figure 1: Phylogenetic analysis of TBCC domain-containing proteins from a range of eukaryotic organisms. A maximum likelihood tree was inferred from alignments using the Metropoliscoupled Markov chain Monte Carlo method implemented by the program FM 3.0b4 (46). Clade 1 (blue) contains canonical TBCC, clade 2 (green) only contains proteins from organisms that contain flagella/cilia and clade 3 (orange) contains atypical TBCC. Genus names are Homo sapiens (HS), Tetraodon nigroviridis (TN), Drosophila melanogaster (DM), Caenorhabditis elegans (CE), Strongylocentrotus purpuratus (SPO), Arabidopsis thaliana (AT), Tetrahymena thermophila (TT), Trypanosoma brucei (TB), Leishmania major (LM), Chlamydomonas reinhardtii (CR), Schizosaccharomyces pombe (SP), Toxoplasma gondii (TG), Plasmodium falciparum (PF) and Giardia lamblia (GL). Posterior probabilities are shown next to the respective branches. See Table S1 for details of the genome projects and references. such as mitosis. In control trypanosomes, mitosis initiates when the new flagellum length is approximately $10 \mu \mathrm{m}$ (Figure 2D), and during the subsequent $M$ phase, the new flagellum continues to elongate to over $15 \mu \mathrm{m}$ as the mitotic spindle elongates (to approximately $8 \mu \mathrm{m}$ ). In contrast, although mitotic spindle microtubules elongate
A
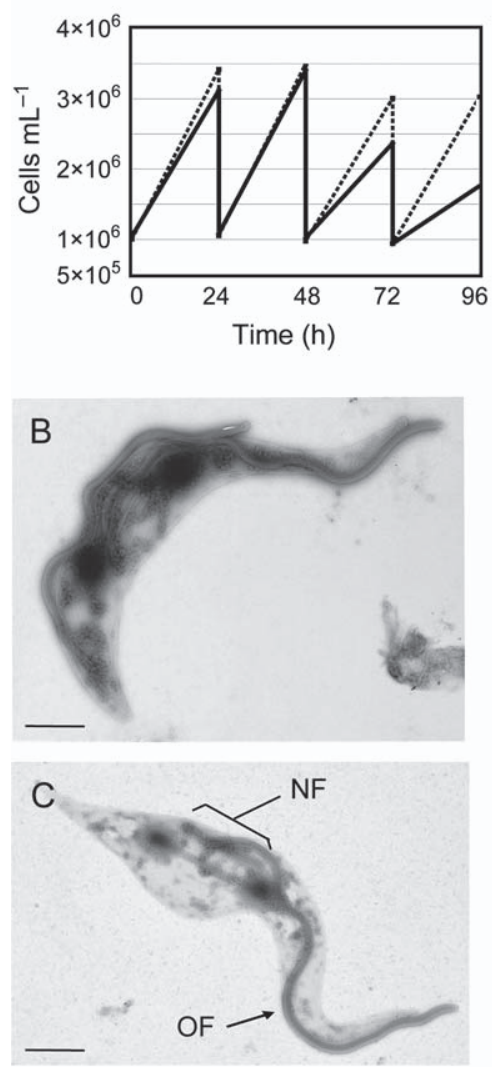

D

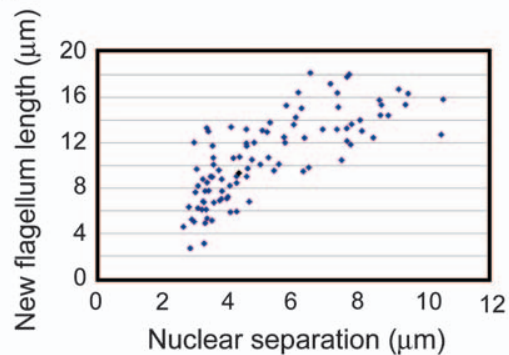

E
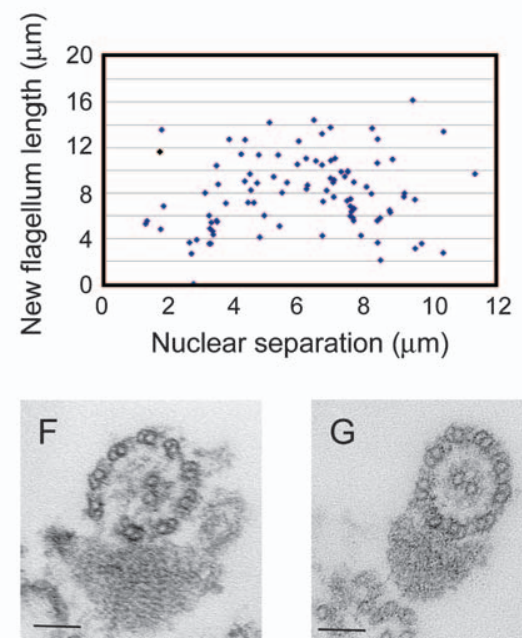

Figure 2: RNA interference-mediated ablation of TbRP2 results in axonemalspecific defects in microtubule assembly. A) Graph showing growth of TbRP2-RNAi induced (black line) and non-induced (dotted line) procyclic trypanosome cell lines. B,C) Transmission electron micrographs of negatively stained whole-mount cytoskeletons from wild-type and TbRP2-ablated procyclic trypanosomes, respectively. OF Old flagellum and NF - new flagellum. D,E) Graphs showing the cell cycle dependent relationship between length of new flagellum and intranuclear separation in non-induced and TbRP2-ablated procyclic trypanosomes, respectively. F, G) Thin-section electron micrographs of transverse sections through the axoneme of TbRP2 induced and noninduced trypanosomes, respectively, showing aberrant axonemal microtubules in the TbRP2 induced cell line. Scale bar in panels $B$ and $C, 2 \mu \mathrm{m}$; scale bar in panels $F$ and $\mathrm{G}, 100 \mathrm{~nm}$. 
as normal in TbRP2-RNAi induced trypanosomes, the new flagellum remains abnormally short (Figure 2E).

This lack of a mitotic defect is significant, as it indicates that spindle microtubule formation is not adversely affected by TbRP2 ablation. Careful examination of the trypanosome cytoskeleton shown in Figure 2C also demonstrates the integrity of sub-pellicular microtubules in the cell body is unaffected. However, a detailed and extensive examination of axonemal ultrastructure in these TbRP2ablated cells reveals that approximately $36 \%$ of axonemes display a range of structural abnormalities; including missing or mis-aligned central pair microtubules and/or disruptions to outer doublet microtubules (Figure 2F). In contrast, a canonical 9+2 axonemal microtubule configuration was observed in non-induced trypanosomes (Figure $2 \mathrm{G}$ ). These results demonstrate that TbRP2 ablation specifically compromises axonemal microtubule formation in the flagellum, without affecting other microtubule populations within the cell; a finding fully consistent with our bioinformatics predictions. It is significant that the ultrastructure of the paraflagellar rod (PFR) is unaffected in these flagella [the PFR is an extra-axonemal structure within the trypanosome flagellum (20)], as this establishes that TbRP2 ablation has specific effects on axonemal microtubule formation rather than a more generalized effect on IFT processes.

\section{TbRP2 specifically interacts with $\alpha$-tubulin at transitional fibres radiating from the mature basal body}

To address the question of how TbRP2 fulfils this flagellumspecific role, we generated trypanosome cell lines expressing a TbRP2:green fluorescent protein (GFP) fusion protein. These studies demonstrated specific localization of TbRP2: GFP at the mature basal body subtending the flagellum (Figure 3A). Importantly, this localization was maintained on detergent-extracted cytoskeletons, indicating that TbRP2 protein is tightly associated with the basal body. At this locality, TbRP2 would be ideally positioned to act in a flagellum-specific capacity, assessing the GTPase competency of native tubulin heterodimer prior to allowing its IFTmediated transport into the cilium/flagellum compartment.

To provide experimental evidence in support of this proposal, we asked whether it was possible to visualize a specific interaction between TbRP2 and tubulin protein at the basal body. The antibody YL1/2 [specific for a carboxyl-tyrosinated form of $\alpha$-tubulin (21)], proved informative in this regard. In many eukaryotes, $\alpha$-tubulin is synthesized with a C-terminal tyrosine residue, but this tyrosine residue is removed following microtubule assembly (reviewed in 22). As new microtubules are tyrosinated but become progressively detyrosinated over time, YL1/2 is regarded as a specific marker for new microtubules. A highly characteristic labelling pattern has previously been reported for YL1/2 on trypanosome cells; tyrosinated $\alpha$-tubulin is restricted to microtubules within the posterior third of the cell body, the axoneme of the new flagellum and the basal bodies subtending new and old flagella $(23,24)$. However, labelling of the trypanosome basal bodies is rather puzzling because basal body microtubules will have been formed at least within the previous cell cycle and in many cases, several generations before that.

We therefore re-investigated this and determined that whilst the YL1/2 labelling presented as two discrete punctuate dots, these did not represent the basal body and probasal body but were specifically associated with the mature basal body (Figure 3B). In colocalization studies, we demonstrate that this $Y L 1 / 2$ staining actually colocalizes with TbRP2:GFP in a distinct ring-shaped structure around the mature basal body (Figure 3C-E). The 'two-dot' pattern emerges when the ring is visualized from the side. Subsequent immunogold electron microscopy experiments confirmed this view and revealed that YL1/2 specifically labels transitional fibres radiating from the mature basal body (Figure 3F). In Figure 3G, colloidal gold particles are clearly evident on transitional fibres radiating from the mature basal body subtending the flagellum, while the immature probasal body (lying orthogonal to the mature basal body in this figure) is $Y L 1 / 2$ negative. Later in the trypanosome cell cycle when both basal bodies are mature (as evidenced by their possession of a transition zone, basal plate and axoneme nucleation), both are $\mathrm{YL} 1 / 2$ positive (Figure $3 \mathrm{H}$ ). The acquisition of $\mathrm{YL1}$ / 2 reactivity through the trypanosome cell cycle can be also visualized by immunofluorescence microscopy (Figure 3I$\mathrm{K})$. Figure $3 \mathrm{I}$ and $\mathrm{J}$ likely represent intermediate stages in the cell cycle, in which acquisition of YL1/2 reactivity is associated with probasal body elongation and nucleation of the new flagellum. This is an important observation as it indicates that an aggregation of tyrosinated $\alpha$-tubulin forms around the basal body as it matures to nucleate the formation of a new flagellum. It should also be noted that the presence of YL1/2 labelling on basal bodies subtending both the old and new flagella suggests a continuing need for tubulin turnover within the microtubule axoneme.

It should be emphasized that we are not suggesting that transitional fibres are constructed from $\alpha$-tubulin; rather that YL1/2 detects a pool of unpolymerized tyrosinated $\alpha$-tubulin recruited to the basal body and awaiting transport into the flagellar compartment by IFT. However, as YL1/2 labelling is retained on whole cells, cytoskeletons and isolated flagella, this pool of unpolymerized tyrosinated $\alpha$-tubulin must be stably associated with the transitional fibres. Significantly following ablation of TbRP2, YL1/2 staining of the basal body is lost (compare basal body staining in Figure $3 \mathrm{~L}$ and M) suggesting not only that TbRP2 interacts with tyrosinated $\alpha$-tubulin, but also that it plays a critical role in the recruitment and/or retention of tubulin at the transitional fibres. In contrast, YL1/2 labelling of the cell body remains intense, confirming that the incorporation of tyrosinated $\alpha$-tubulin into sub-pellicular microtubules is unaffected in TbRP2-ablated cells. 

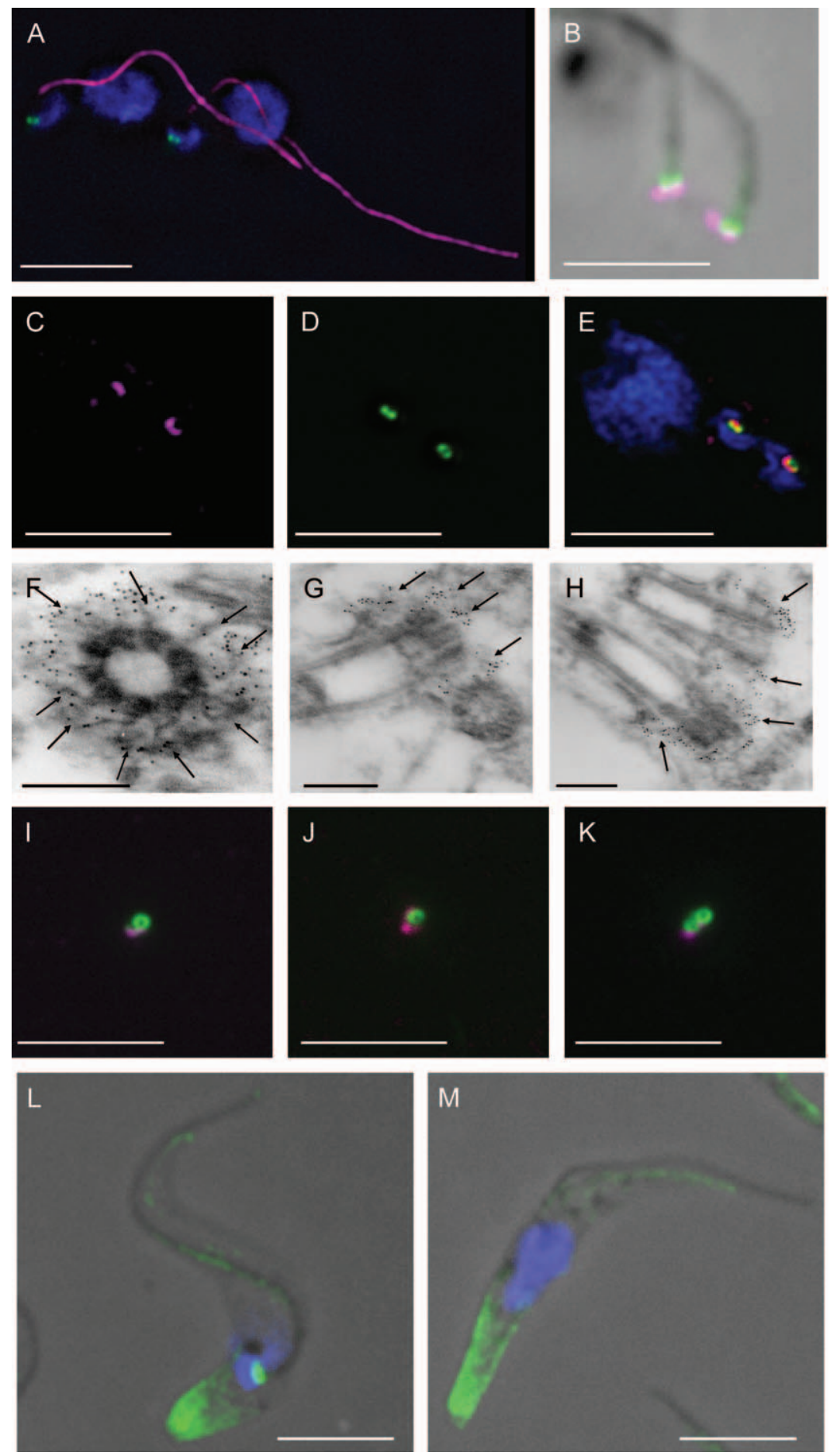

Figure 3: TbRP2 and tyrosinated $\alpha$-tubulin specifically colocalize at transitional fibres radiating from the mature basal body. A) Immunofluorescence image of a $2 \mathrm{~K} 2 \mathrm{~N}$ procyclic trypanosome cell expressing the TbRP2:GFP fusion protein (green) colabelled with the anti-PFR antibody, L8C4 (magenta). Nuclear and kinetoplast DNA are labelled with 4', 6-diamidino-2-phenylindole (blue). B) Immunofluorescence image of purified flagella co-labelled with the anti-basal body antibody, BBA4 (magenta) and the anti-tyrosinated $\alpha$-tubulin antibody, YL1/2 (green) overlaid onto a phase contrast image. C-E) Immunofluorescence images of a $2 \mathrm{~K} 2 \mathrm{~N}$ procyclic trypanosome expressing the TbRP2:GFP fusion protein (green) co-labelled with YL1/2 (magenta). Nuclear and kinetoplast DNA are labelled with DAPI (blue). F-H) Thin-section electron micrographs showing YL1/2 labelling of transitional fibres radiating from a mature basal body subtending a flagellum. The immature probasal body shown in figure $3 G$ is $Y L 1 / 2$ negative; however, by the time the new basal body has matured and nucleated a flagellum (3H) YL1/2 reactivity has been acquired. I-K) Immunofluorescence images of purified flagella isolated from procyclic trypanosomes at various stages in the cell cycle. Flagella are co-labelled with the anti-basal body antibody, BBA4 (magenta) and the anti-tyrosinated $\alpha$-tubulin antibody, YL1/2 (green). Nuclear and kinetoplast DNA are labelled with DAPI (blue). L-M) Immunofluorescence images of non-induced $(L)$ and TbRP2-ablated (M) procyclic trypanosomes labelled with the anti-tyrosinated $\alpha$-tubulin antibody, YL1/2 (green) overlaid onto phase contrast images. Nuclear and kinetoplast DNA are labelled with DAPI (blue). Scale bars: Panels A-E, $5 \mu \mathrm{m}$; Panels $\mathrm{F}-\mathrm{H}, 200 \mathrm{~nm}$; Panels I-M, $5 \mu \mathrm{m}$.

\section{Discussion}

Our study resolves a long-standing conundrum as to why YL1/2 (a reliable and documented marker for new microtubules) apparently labelled basal bodies given their microtubule maturation cycle (25). Our studies demonstrate that YL1/2 detects an aggregation of unpolymerized tubulin rather than the basal body microtubules themselves.
In most ciliated/flagellated eukaryotic cells, the formation of a cilium/flagellum is dependent upon IFT, an evolutionarily conserved transport mechanism that moves proteins bi-directionally along the length of the flagellum. The basal body is pivotal to IFT, with transitional fibres radiating from the mature basal body acting as the docking site for IFT motor proteins at the base of the cilium/flagellum (reviewed in 26). These transitional fibres provide a critical 


\section{Stephan et al.}

interface between cytoplasmic and cilium/flagellum compartments and are proposed to regulate IFT particle entry into the cilium/flagellum (11). However, data from this current study further extends the role of the basal body in cilium/flagellum assembly and suggests that a specific isoform of TBCC embedded at these transitional fibres acts to influence the 'quality' of tubulin heterodimers destined for axonemal incorporation.

Previous biochemical studies suggest functional overlap between RP2 and TBCC (17), whereby RP2 acts to stimulate the GTPase activity of native tubulin heterodimers but does not function in the generation of $\alpha / \beta$-tubulin heterodimer from tubulin monomers (17). This biochemical evidence indicates that RP2 is not part of a canonical tubulin-folding pathway but rather provides specific functions related to assessing GTPase activity of tubulin heterodimer. Our bioinformatics analysis further reveals that RP2-like proteins are phylogenetically restricted to ciliated/ flagellated organisms and suggests that this protein has evolved to deal with the specific demands of tubulin provision in a cilium/flagellum context; a proposal strongly supported by our experimental RNAi studies.

Despite clear biochemical evidence indicating a role for RP2 in microtubule dynamics (17), the role of RP2 in vivo remains a matter of debate because it has recently been suggested that RP2 exhibits $3^{\prime}$ to $5^{\prime}$ exonuclease activity and relocates to the nucleus in response to DNA damage (19). While our study does not address this proposed exonuclease role, it provides direct in vivo evidence that axonemal microtubule formation is defective following ablation of TbRP2. This result is consistent with previously published in vitro biochemical evidence indicating a role for RP2 in tubulin processing.

However, in this processing context, it will be important to determine whether RP2 acts solely upon newly translated tubulin heterodimers or also upon re-recycling tubulin heterodimers previously assembled into other microtubule structures. Elegant experimental studies carried out on the biflagellate algal cell Chlamydomonas, suggest that reutilization of tubulin heterodimer may occur in flagellated eukaryotic cells (27). These studies demonstrated that following removal of one of its two flagella, Chlamydomonas shortens the remaining flagellum and simultaneously regenerates the amputated flagellum. This pattern of flagellum elongation and shortening was not affected by cycloheximide (a treatment that blocks new protein synthesis). In addition to accessing pre-existing cytoplasmic pools of tubulin, it is possible that these cells also re-utilize tubulin heterodimers, previously incorporated in the axoneme of the shortening flagellum. Our bioinformatics analysis reveals that Chlamydomonas encodes two RP2like proteins, one of which at least is present in the Chlamydomonas flagellar proteome (5). We suggest that elucidation of the role of these Chlamydomonas RP2-like proteins in tubulin recycling will be informative.
As ablation of TbRP2 results exclusively in axonemal microtubule defects, our data provide strong evidence that ciliary defects observed in X-linked RP2 arise from aberrant tubulin processing. Interestingly, the $C$. elegans protein K08D12.2 (a RP2-like protein also found in clade 2) also localizes to the basal body of ciliated neuronal cells; indeed, the ring-like localization pattern reported for K08D12.2 (28) is remarkably consistent with our localization of TbRP2:GFP.

However, there is an important caveat to this argument; as the human RP2 has not been reported to localize to the basal body but rather to the plasma membrane because of dual acyl modification of the N-terminus of the protein (29). If RP2 function in sensory neurons relates solely to tubulin processing, then one has to rehearse the argument that $\mathrm{N}$-acylated RP2 proteins can fulfil this protein-processing function from the plasma membrane. A clue to how this might occur comes from studies on human retinal cells which show that membrane bound RP2 interacts with microtubule associated proteins such as ADP-ribosylation factor-like 3 (ARL3). It is suggested that this interaction links the membrane and microtubule cytoskeleton and acts to regulate membrane traffic and/or cell signalling (47).

In this study, we demonstrate that TbRP2 (a flagellumspecific isoform of TBCC) is exclusively localized at transitional fibres radiating from the trypanosome basal body. As RNAi-mediated ablation of TbRP2 results in the specific loss of tyrosinated $\alpha$-tubulin from the basal body, we suggest a specific interaction occurs between these two proteins. As transitional fibres demarcate the boundary between the cytoplasm and the environmentally distinct flagellum compartment, we propose that TbRP2 establishes a 'quality control gateway' to assess the GTPase proficiency of tubulin heterodimer prior to IFT-mediated transport into the cilium/flagellum.

One can also rehearse another role for a tubulin quality control gateway at the basal body, namely that it may prevent or select for entry of post-translationally modified tubulin dimers into the flagellum compartment. If this is true, then enzymatic functions associated with the reversible tubulin post-translational modifications (tyrosination, acetylation and glutamylation) might also be expected to localize to the basal body. In trypanosomes, recycled tubulin would need to be retyrosinated through the action of a tubulin tyrosine ligase (TTL) $(24,30)$. Can the basal body also act as a localized factory to retyrosinate $\alpha$-tubulin previously incorporated into microtubules? Several TTLlike proteins and other tubulin modification enzymes (such as polyglutamylases) are encoded within the trypanosome genome (31) and one might predict that these proteins may also be localized to the trypanosome basal body.

Finally, we suggest that aberrant protein processing at the cilium/flagellum basal body may underpin a wide-range of 
human ciliopathies, as we note that mutations in BBS6 and BBS10 (proteins with homology to $\alpha$ and $\zeta$ subunits of cytosolic chaperonin) also result in the development of Bardet-Biedl syndrome (a condition characterized by genital, renal and retinal abnormalities resulting from abnormal basal body function and cilium formation) $(32,33)$. The establishment of a 'quality control' gateway at the basal body may therefore represent a widespread mechanism by which ciliated/flagellated cells ensure the fidelity of the microtubule axoneme.

\section{Materials and Methods}

\section{Trypanosome culture and generation of $\mathrm{T}$. brucei RNAi cell lines}

Procyclic T. brucei cells were routinely grown in semi-defined medium-79 media supplemented with $15 \%$ fetal calf serum as previously described (34). Trypanosome cell growth was monitored using a CASY $1{ }^{\circledR}$ cell counter and analyser system (Schärfe System $\mathrm{GmbH}$ ) with cultures diluted on a daily basis to maintain a density of between $1 \times 10^{6}$ and $8 \times 10^{6} \mathrm{cell} / \mathrm{s} / \mathrm{mL}$. A 577 nucleotide fragment of the T. brucei, Tb10.61.2870 coding sequence was amplified by polymerase chain reaction (PCR) using gene-specific primers 2870RNAiF (5'- GCAGCTCGAGATAACCCTGCACATCTTCGC-3') and 2870RNAiR (5'-GCAGGGATCCGAGAATCGTTCTCGTCCTCG-3'), and cloned into the T. brucei RNAi vector, p2T7-177 (35). The resulting construct was linearized with Notl, transfected into the procyclic 29-13 cell line and positive transformants were selected using $2.5 \mu \mathrm{g} / \mathrm{mL}$ phleomycin, $15 \mu \mathrm{g} / \mathrm{mL}$ G418 and $50 \mu \mathrm{g} / \mathrm{mL}$ hygromycin as previously described (36).

\section{Induction of RNAi and phenotypic analysis}

Trypanosome cultures (grown in drug-free media for $24 \mathrm{~h}$ prior to initiation of RNAi studies) were diluted to $1 \times 10^{6}$ cells $/ \mathrm{mL}$ and doxycycline was added to a final concentration of $1 \mu \mathrm{g} / \mathrm{mL}$. Every $24 \mathrm{~h}$, cell cultures were counted and diluted back to a density of $1 \times 10^{6}$ cells $/ \mathrm{mL}$ with fresh doxycycline being added to the culture medium.

\section{Green fluorescent protein tagging of the endogenous Tb10.61.2870 open reading frame}

DNA fragments from the $3^{\prime}$ end of the Tb10.61.2870 open reading frame (ORF) sequence (minus the TAG stop codon) and the immediate $3^{\prime}$ untranslated region (UTR) downstream of the TAG stop codon were amplified by PCR using the primer combinations Tb10.61.2870/orfFTb10.61.2870/orfR and Tb10.61.2870/utrF-Tb10.61.2870/utrR, respectively (primer sequences available on request). Resultant DNA fragments were simultaneously ligated into pET-HYG-GFP (Devaux et al., 2007). The resultant pET-GFP.2870 vector was subsequently linearized with Xhol and transfected into procyclic T. brucei 427 strain cells. Positive cells were selected using media containing $20 \mu \mathrm{g} / \mathrm{mL}$ hygromycin as described (37)

\section{Immunofluorescence studies}

Procyclic trypanosomes (both wild type and cells expressing GFP fusion proteins) were prepared for microscopy essentially as previously described (38). Slides were incubated with selected primary antibodies YL1/2 (39), L8C4 (40) or BBA4 (41). Slides were examined either on a DeltaVision RT microscope equipped with a Hamamatsu CCD camera and images processed in SOFTWoRx (Applied Precision) or on a Zeiss Axioplan 2 microscope equipped with a CCD camera controlled by METAMORPH software (Universal Imaging) and processed in METAMORPH. All images were subsequently processed using Adobe Photoshop (Adobe).

\section{Electron microscopy and immunogold labelling}

Trypanosomes were harvested by centrifugation $(800 \times \boldsymbol{g}, 10 \mathrm{~min})$, fixed in $2 \%$ paraformaldehyde, $2 \%$ glutaraldehyde and $0.2 \%$ picric acid in $100 \mathrm{~mm}$ phosphate buffer ( $\mathrm{pH} 7.2$ ), post-fixed and processed for transmission electron microscopy as described previously (42). Preparation of negatively stained whole mount cytoskeletons for transmission electron microscopy was carried out using previously established methods $(42,43)$. For pre-embedding, immunogold labelling cells were fixed with $8 \%$ formaldehyde in $100 \mathrm{~mm}$ phosphate buffer $(\mathrm{pH} 7.0)$ with $0.5 \%$ Triton-X-100 for $\sim 2 \mathrm{~min}$. The cells were gently pelleted and briefly quenched $(2 \times 30$ seconds) with PBS containing $1 \%$ BSA, 50 mm glycine and $0.1 \%$ Tween- 20 followed by a brief wash in PBS blocking solution (PBS containing 1\% BSA and $0.1 \%$ Tween-20). Samples were incubated with YL1/2 (diluted 1:2 in PBS blocking buffer) for $2 \mathrm{~h}$ at room temperature. After washing with blocking buffer $(3 \times 10 \mathrm{~min})$, the samples were incubated with a goat anti-rat antibody conjugated with $5 \mathrm{~nm}$ gold (1:50 dilution with PBS blocking buffer), washed with blocker $(2 \times 10 \mathrm{~min})$ and the samples were fixed and processed as described above. Ultra-thin ( 70 nm thick) sections were examined unstained in a Philips 400 electron microscope.

\section{Bioinformatics analyses}

Tubulin cofactor $\mathrm{C}$ homologues in diverse eukaryotic organisms were identified by an iterative profile-based searching technique using the Pfam TBCC domain seed alignment (44). A Hidden Markov Model (HMM) was used to search the genome databases of a range of eukaryotic organisms (Table S1) essentially as detailed in (45).

\section{Acknowledgments}

This work was supported by grants from the Biotechnology and Biological Sciences Research Council (BBSRC), The Royal Society, the E. P. Abraham Trust and the Wellcome Trust. K. G. is a Wellcome Trust Principal Research Fellow. We thank current members of our respective laboratories for their valuable conversations on this work and also the Genome Projects used in this study.

\section{Supplementary Material}

Table S1: Sources and versions of genomic data used in this paper

Supplemental materials are available as part of the online article at http:// www.blackwell-synergy.com

\section{References}

1. Bisgrove BW, Yost HJ. The roles of cilia in developmental disorders and disease. Development 2006;133:4131-4143.

2. Broadhead R, Dawe HR, Farr H, Griffiths S, Hart SR, Portman N, Shaw MK, Ginger ML, Gaskell SJ, McKean PG, Gull K. Flagellar motility is required for the viability of the bloodstream trypanosome. Nature 2006;440:224-227.

3. Li J, Gerdes J, Haycraft C, Fan Y, Teslovich T, May-Simera H, Li H, Blacque O, Li L, Leitch C, Lewis R, Green J, Parfrey P, Leroux M, Davidson $W$ et al. Comparative genomics identifies a flagellar and basal body proteome that includes the BBS5 human disease gene. Cell 2004;117:541-552.

4. Ostrowski LE, Blackburn K, Radde KM, Moyer MB, Schlatzer DM, Moseley A, Boucher RC. A proteomic analysis of human cilia: identification of novel components. Mol Cell Proteomics 2002;1:451-465.

5. Pazour GJ, Agrin N, Leszyk J, Witman GB. Proteomic analysis of a eukaryotic cilium. J Cell Biol 2005;170:103-113.

6. Johnson K, Rosenbaum J. Polarity of flagellar assembly in Chlamydomonas. J Cell Biol 1992;119:1605-1611. 


\section{Stephan et al.}

7. Briggs LJ, Davidge JA, Wickstead B, Ginger ML, Gull K. More than one way to build a flagellum: comparative genomics of parasitic protozoa. Curr Biol 2004;14:R611-R612.

8. Han YG, Kwok BH, Kernan MJ. Intraflagellar transport is required in Drosophila to differentiate sensory cilia but not sperm. Curr Biol 2003;13:1679-1686.

9. Scholey J. Intraflagellar transport. Annu Rev Cell Dev Biol 2003; 19:423-443.

10. Deane J, Cole D, Seeley E, Diener D, Rosenbaum J. Localization of intraflagellar transport protein IFT52 identifies basal body transitional fibers as the docking site for IFT particles. Curr Biol 2001;11:1586-1590.

11. Dentler W. Intraflagellar transport (IFT) during assembly and disassembly of Chlamydomonas flagella. J Cell Biol 2005;170:649-659.

12. Lewis SA, Tian G, Vainberg IE, Cowan NJ. Chaperonin-mediated folding of actin and tubulin. J Cell Biol 1996;132:1-4.

13. Tian G, Bhamidipati A, Cowan NJ, Lewis SA. Tubulin folding cofactors as GTPase-activating proteins. GTP hydrolysis and the assembly of the alpha/beta-tubulin heterodimer. J Biol Chem 1999;274:24054-24058.

14. Tian G, Huang Y, Rommelaere H, Vandekerckhove J, Ampe C, Cowan NJ. Pathway leading to correctly folded beta-tubulin. Cell 1996;86:287-296.

15. Tian G, Lewis SA, Feierbach B, Stearns T, Rommelaere H, Ampe C, Cowan NJ. Tubulin subunits exist in an activated conformational state generated and maintained by protein cofactors. J Cell Biol 1997;138:821-832.

16. Hamel C. Retinitis pigmentosa. Orphanet J Rare Dis 2006;1:40.

17. Bartolini F, Bhamidipati A, Thomas S, Schwahn U, Lewis SA, Cowan NJ. Functional overlap between retinitis pigmentosa 2 protein and the tubulin-specific chaperone cofactor C. J Biol Chem 2002;277:14629-14634.

18. Briggs LJ, McKean PG, Baines A, Moreira-Leite F, Davidge JA, Vaughan S, Gull K. The flagella connector of Trypanosoma brucei: an unusual mobile transmembrane junction. J Cell Sci 2004;117:1641-1651.

19. Yoon JH, Qiu J, Cai S, Chen Y, Cheetham ME, Shen B, Pfeifer GP. The retinitis pigmentosa-mutated $\mathrm{RP} 2$ protein exhibits exonuclease activity and translocates to the nucleus in response to DNA damage. Exp Cell Res 2006;312:1323-1334.

20. Bastin P, Matthews K, Gull K. The paraflagellar rod of kinetoplastida: solved and unsolved questions. Parasitol Today 1996;12:302-307.

21. Wehland J, Schroder HC, Weber K. Amino acid sequence requirements in the epitope recognized by the alpha-tubulin-specific rat monoclonal antibody YL1/2. EMBO J 1984;3:1295-1300.

22. Idriss $\mathrm{H}$. Man to trypanosome: the tubulin tyrosination/detyrosination cycle revisited. Cell Motil Cytoskeleton 2000;45:173-184.

23. Sasse R, Gull K. Tubulin post-translational modifications and the construction of microtubular organelles in Trypanosoma brucei. J Cell Sci 1988;90:577-589.

24. Sherwin T, Schneider A, Sasse R, Seebeck T, Gull K. Distinct localisation and cell cycle dependence of $\mathrm{COOH}$ terminally tyrosinolated a-tubulin in the microtubules of Trypanosoma brucei brucei. J Cell Biol 1987;104:439-446.

25. Kochanski RS, Borisy GG. Mode of centriole duplication and distribution. J Cell Biol 1990;110:1599-1605.

26. Rosenbaum J, Witman G. Intraflagellar transport. Nat Rev Mol Cell Biol 2002;3:813-825.

27. Rosenbaum J, Moulder J, Ringo, D. Flagellar elongation and shortening in Chlamydomonas. The use of cycloheximide and colchicine to study the synthesis and assembly of flagellar proteins. J Cell Biol 1969;41:600-619.

28. Blacque OE, Perens EA, Boroevich KA, Inglis PN, Li C, Warner A, Khattra J, Holt RA, Ou G, Mah AK, McKay SJ, Huang P, Swoboda P, Jones SJ, Marra MA et al. Functional genomics of the cilium, a sensory organelle. Curr Biol 2005;15:935-941.

29. Chapple JP, Hardcastle AJ, Grayson C, Willison KR, Cheetham, ME. Delineation of the plasma membrane targeting domain of the $\mathrm{X}$-linked retinitis pigmentosa protein RP2. Invest Ophthalmol Vis Sci 2002;43:2015-2020.
30. Gallo JM, Precigout E. Tubulin expression in trypanosomes. Biol Cell 1988;64:137-143.

31. Janke C, Rogowski K, Wloga D, Regnard C, Kajava AV, Strub JM, Temurak N, van Dijk J, Boucher D, van Dorsselaer A, Suryavanshi S, Gaertig J, Edde B. Tubulin polyglutamylase enzymes are members of the TTL domain protein family. Science 2005;308:1758-1762.

32. Kim JC, Ou YY, Badano JL, Esmail MA, Leitch CC, Fiedrich E, Beales PL, Archibald JM, Katsanis N, Rattner JB, Leroux MR. MKKS/BBS6, a divergent chaperonin-like protein linked to the obesity disorder Bardet-Biedl syndrome, is a novel centrosomal component required for cytokinesis. J Cell Sci 2005;118:1007-1020.

33. Stoetzel C, Laurier V, Davis EE, Muller J, Rix S, Badano JL, Leitch CC, Salem N, Chouery E, Corbani S, Jalk N, Vicaire S, Sarda P, Hamel C, Lacombe D et al. BBS10 encodes a vertebrate-specific chaperonin-like protein and is a major BBS locus. Nat Genet 2006;38:521-524.

34. McKean PG, Baines A, Vaughan S, Gull K. Gamma-tubulin functions in the nucleation of a discrete subset of microtubules in the eukaryotic flagellum. Curr Biol 2003;13:598-602.

35. Wickstead B, Ersfeld K, Gull K. Targeting of a tetracycline-inducible expression system to the transcriptionally silent minichromosomes of Trypanosoma brucei. Mol Biochem Parasitol 2002;125:211-216.

36. Gadelha C, Wickstead B, McKean PG, Gull K. Basal body and flagellum mutants reveal a rotational constraint of the central pair microtubules in the axonemes of trypanosomes. J Cell Sci 2006;119:2405-2413.

37. Devaux S, Kelly S, Lecordier L, Wickstead B, Perez-Morga D, Pays E, Vanhamme L, Gull K. Diversification of function by different isoforms of conventionally shared RNA polymerase subunits. Mol Biol Cell 2007; 18:1293-1301.

38. Davidge JA, Chambers E, Dickinson HA, Towers K, Ginger ML, McKean PG, Gull K. Trypanosome IFT mutants provide insight into the motor location for mobility of the flagella connector and flagellar membrane formation. J Cell Sci 2006;119:3935-3943.

39. Kilmartin JV, Wright B, Milstein C. Rat monoclonal anti tubulin antibodies derived by using a new nonsecreting rat cell line. J Cell Biol 1982;93:576-582.

40. Kohl L, Sherwin T, Gull K. Assembly of the paraflagellar rod and the flagellum attachment zone complex during the Trypanosoma bruceicell cycle. J Eukaryot Microbiol 1999;46:105-109.

41. Woodward R, Carden MJ, Gull K. Immunological characterization of cytoskeletal proteins associated with the basal body, axoneme and flagellum attachment zone of Trypanosoma brucei. Parasitology 1995;111:77-85.

42. Sherwin T, Gull K. The cell division cycle of Trypanosoma brucei brucei: timing of event markers and cytoskeletal modulations. Philos Trans R Soc Lond B Biol Sci 1989;323:573-588.

43. Moreira-Leite F, Sherwin T, Kohl L, Gull K. A trypanosome structure involved in transmitting cytoplasmic information during cell division. Science 2001;294:610-612.

44. Bateman A, Coin L, Durbin R, Finn RD, Hollich V, Griffiths-Jones S, Khanna A, Marshall M, Moxon S, Sonnhammer EL, Studholme DJ, Yeats C, Eddy SR. The Pfam protein families database. Nucleic Acids Res 2004;32:D138-D141.

45. Kelly S, Wickstead B, Gull K. An in silico analysis of trypanosomatid RNA polymerases: insights into their unusual transcription. Biochem Soc Trans 2005;33:1435-1437.

46. Huelsenbeck J, Ronquist F. MRBAYES: Bayesian interence of phylogenetic trees. Bioinformatics 2001;17:754-755.

47. Grayson C, Bartolini F, Chapple JP, Willison KR, Bhamidipati A, Lewis SA, Luthert PJ, Hardcastle AJ, Cowan NJ, Cheetham ME. Localization in the human retina of the X-linked retinitis pigmentosa protein RP2, its homologue cofactor $\mathrm{C}$ and the RP2 interacting protein Arl3. Hum Mol Genet 2002;11:3065-3074. 